\title{
E-UNITARY CONGRUENCES ON INVERSE SEMIGROUPS
}

\author{
by N. R. REILLY and W. D. MUNN
}

(Received 10 January, 1975)

By an $E$-unitary inverse semigroup we mean an inverse semigroup in which the semilattice is a unitary subset. Such semigroups, elsewhere called "proper" or "reduced" inverse semigroups, have been the object of much recent study. Free inverse semigroups form a subclass of particular interest.

An important structure theorem for $E$-unitary inverse semigroups has been obtained by McAlister $[4,5]$. From a triple $(G, \mathscr{X}, \mathscr{Y})$ consisting of a group $G$, a partially ordered set $\mathscr{X}$ and a subset $\mathscr{Y}$ of $\mathscr{X}$, satisfying certain conditions, he constructs an $E$-unitary inverse semigroup $P(G, \mathscr{X}, \mathscr{Y})$. A semigroup of this type is called a $P$-semigroup. The structure theorem states that every $E$-unitary inverse semigroup is, to within isomorphism, of this form. A second theorem asserts that every inverse semigroup is isomorphic to a quotient of a $P$ semigroup by an idempotent-separating congruence. We refer below to these results as McAlister's Theorems A and B respectively. A triple $(G, \mathscr{X}, \mathscr{Y})$ of the type used to construct a $P$-semigroup is here termed a "McAlister triple". It is shown further, in [5], that there is essentially only one such triple corresponding to a given $E$-unitary inverse semigroup.

A congruence $\rho$ on an inverse semigroup $S$ is called an $E$-unitary congruence if and only if $S / \rho$ is an $E$-unitary inverse semigroup. The main purpose of this paper is to provide some results on such congruences.

In $\S 1$, we give an account of some basic properties of congruences on inverse semigroups, with the emphasis on group congruences, idempotent-separating congruences and idempotentdetermined congruences (defined in [2]; see below). It is noted that an idempotent-determined congruence on an $E$-unitary inverse semigroup is necessarily $E$-unitary. In $\$ 2$, properties of $P$-semigroups are reviewed and, for a McAlister triple $(G, \mathscr{X}, \mathscr{Y})$, we introduce and examine two further concepts: $T$-congruences on $\mathscr{X}$ and the kernel of $(G, \mathscr{X}, \mathscr{Y})$.

Idempotent-determined congruences and idempotent-separating congruences on $P$ semigroups are investigated in $\$ 3$ and $\$ 4$ respectively, with the techniques developed in $\$ 2$. In particular, it is shown in Theorem 3.2 that every quotient of a $P$-semigroup by an idempotentdetermined congruence is isomorphic to a $P$-semigroup, the McAlister triple for the quotient being derived explicitly from that of the given $P$-semigroup. Theorem 4.5 provides a similar result for the quotient of a $P$-semigroup by an $E$-unitary idempotent-separating congruence.

These results are applied in $\$ 5$ to the case of a free inverse semigroup to yield a new proof of McAlister's Theorems A and B. We also define the notion of a quasi-free inverse semigroup and establish some basic properties of such a system.

The final section, $\S 6$, is devoted to a brief study of the set $\Lambda^{*}$ of all $E$-unitary congruences on an arbitrary inverse semigroup $S$. It is shown that $\Lambda^{*}$ is a complete lattice with respect to inclusion, but not, in general, a sublattice of the lattice $\Lambda(S)$ of all congruences on $S$. Certain sublattices of $\Lambda^{*}$, and the relationship between $\Lambda^{*}$ and the $\theta$-classes of $\Lambda(S)$ (in the sense of [17]) are also discussed. 
1. Preliminaries. Throughout the paper the basic terminology and notation will be that of [1]:

We begin with some remarks on sets. Let $X$ be a nonempty set. The identity relation on $X$ will be denoted by $l_{X}$. For any equivalence $\rho$ on $X$ the $\rho$-class containing $x \in X$ will be denoted by $x \rho$ and the natural mapping $x \mapsto x \rho$ from $X$ to $X / \rho$ by $\rho$. If $\rho, \tau$ are equivalences on $X$ such that $\rho \subseteq \tau$ then $\tau / \rho$ will denote the equivalence on $X / \rho$ defined by the rule that $(x \rho, y \rho) \in \tau / \rho$ if and only if $(x, y) \in \tau$.

Now suppose that $\leqslant$ is a partial ordering of $X$. We say that $X$ is lower directed if and only if, for all $x, y \in X$, there exists $z \in X$ such that $z \leqslant x$ and $z \leqslant y$. By a convex subset of $X$ we mean a subset $Y$ such that, for all $y_{1}, y_{2} \in Y$ and all $x \in X, y_{1} \leqslant x \leqslant y_{2}$ implies $x \in Y$. A nonempty subset $Y$ of $X$ is called an ideal of $X$ if and only if, for all $x \in X$ and all $y \in Y, x \leqslant y$ implies $x \in Y$. We call $X$ a lower semilattice if and only if every pair of elements of $X$ has a greatest lower bound with respect to the partial ordering. As is customary, we shall sometimes regard a lower semilattice as a commutative semigroup of idempotents in which the product of two elements is their greatest lower bound; conversely a commutative semigroup $S$ of idempotents is a lower semilattice with respect to the partial ordering $\leqslant$ defined by the rule that $x \leqslant y$ if and only if $x y=x(x, y \in S)$. In $\$ 2$ we shall be concerned with a partially ordered set $\mathscr{X}$ containing an ideal $\mathscr{Y}$ which is also a lower semilattice under the partial ordering of $\mathscr{X}$.

We now review some elementary properties of congruences on inverse semigroups. Where no other reference is given, proofs may be found in [1, Chapter 7]. Let $S$ be an inverse semigroup with semilattice $E$. The set of all congruences on $S$ forms a complete lattice with respect to partial ordering by inclusion: it will be denoted by $\Lambda(S)$. Let $\rho \in \Lambda(S)$. Then, for all $a \in S$,

$$
\left(a, a^{2}\right) \in \rho \Rightarrow(a, e) \in \rho \text { for some } e \in E,
$$

from which it follows that the quotient semigroup $S / \rho$ is again an inverse semigroup. Also, for all $a, b \in S$,

Now let $\tau \in \Lambda(S)$. Then

$$
(a, b) \in \rho \Rightarrow\left(a^{-1}, b^{-1}\right) \in \rho .
$$

$$
\rho \subseteq \tau \Leftrightarrow e \rho \subseteq e \tau \text { for all } e \in E
$$

This shows, in particular, that $\rho=\tau$ if and only if $e \rho=e \tau$ for all $e \in E$. If $\rho \subseteq \tau$ then $\tau / \rho$ is a congruence on $S / \rho$.

By a normal equivalence on $E$ we shall mean an equivalence $\pi$ satisfying the following conditions ([17]):

(i) $(e, f) \in \pi \Rightarrow(e g, f g) \in \pi$ for all $g \in E$,

(ii) $(e, f) \in \pi \Rightarrow\left(x e x^{-1}, x f x^{-1}\right) \in \pi$ for all $x \in S$.

It is easily seen that if $\rho \in \Lambda(S)$ then $\rho \cap(E \times E)$ is a normal equivalence on $E$. Furthermore, the relation $\theta$ defined on $\Lambda(S)$ by

$$
\left(\rho_{1}, \rho_{2}\right) \in \theta \Leftrightarrow \rho_{1} \cap(E \times E)=\rho_{2} \cap(E \times E)
$$

is a congruence on the lattice $\Lambda(S)$ and each $\theta$-class is a complete modular sublattice of $\Lambda(S)$ 
[17, Theorem 5.1]. Now suppose that $\pi$ is any normal equivalence on $E$. Then

$$
\{\rho \in \Lambda(S): \rho \cap(E \times E)=\pi\}
$$

is nonempty [17, Theorem 4.2] and so forms a $\theta$-class of $\Lambda(S)$. In particular this set has a greatest and a least element. The least element is the congruence on $S$ generated by $\pi$ (viewed as a relation on $S)$ and it will be denoted by $\pi^{*}$. We note that, for all $\rho \in \Lambda(S),[\rho \cap(E \times E)]^{*} \subseteq \rho$.

Several special types of congruence on $S$ will be central to our discussion. First, we call $\rho \in \Lambda(S)$ a group congruence if and only if $S / \rho$ is a group. As shown in [8, Theorem 1], $S$ possesses a least group congruence $\sigma$ and this has the following characterisation:

$$
(a, b) \in \sigma \Leftrightarrow e a=e b \text { for some } e \in E .
$$

Since $S / \sigma$ is a group, $E \times E \subseteq \sigma$; that is, $E$ is contained in a single $\sigma$-class. In general, $E$ will not itself be a $\sigma$-class, but we shall be concerned with the case in which it is (see Lemma 1.6 below). It is readily verified that if $\rho \in \Lambda(S)$ is such that $\rho \subseteq \sigma$, and if $\sigma_{1}$ denotes the least group congruence on $S / \rho$, then

$$
\sigma_{1}=\sigma / \rho
$$

The relation $\leqslant$ defined on $S$ by the rule that

$$
a \leqslant b \Leftrightarrow a=e b \text { for some } e \in E
$$

is a partial ordering of $S$ (called the natural partial ordering) [1, Chapter 7]. From (1.4), it can be seen that, under $\leqslant$, each $\sigma$-class of $S$ is lower directed.

Second, we call $\rho \in \Lambda(S)$ an idempotent-separating congruence if and only if each $\rho$-class contains at most one idempotent. A congruence $\rho$ on $S$ is idempotent-separating if and only if $\rho \subseteq \mathscr{H}$. Consequently, there exists a greatest idempotent-separating congruence $\mu$ on $S$, namely the greatest congruence contained in $\mathscr{H}$. Howie [3] has provided a simple characterisation of $\mu$. It can readily be shown that if $\rho, \tau \in \Lambda(S)$ are such that $\rho \subseteq \tau$ and $\rho \cap(E \times E)=$ $\tau \cap(E \times E)$ then $\tau / \rho$ is an idempotent-separating congruence on $S / \rho$. In particular, if $\rho \in \Lambda(S)$ and if $\pi$ denotes $\rho \cap(E \times E)$ then $\pi^{*} \subseteq \rho$ (as remarked above) and $\rho / \pi^{*}$ is an idempotentseparating congruence on $S / \pi^{*}$.

Third, we call $\rho \in \Lambda(S)$ an idempotent-determined congruence on $S$ if and only if each $\rho$ class that contains an idempotent consists entirely of idempotents. Such congruences have been studied by D. G. Green [2].

A subset $A$ of $S$ is termed left unitary if and only if, for all $a \in A$ and all $x \in S, a x \in A$ implies $x \in A$. Right unitary subsets are defined dually. By a unitary subset of $S$ we mean a subset that is both left and right unitary. Suppose that the semilattice $E$ of $S$ is left unitary. Then it is also right unitary; for if $e \in E$ and $x \in S$ are such that $x e \in E$ then $e x^{-1}=(x e)^{-1} \in E$ and so $x^{-1} \in E$, which implies that $x \in E$. This establishes the equivalence of conditions (i) and (ii) of the lemma below. Conditions (ii) and (iii) are clearly equivalent and the equivalence of (iii), (iv) and (v) has been noted elsewhere (for example, see [5, Proposition 1.1]).

LEMma 1.6. The following conditions on an inverse semigroup $S$, with semilattice $E$ and least group congruence $\sigma$, are equivalent: 
(i) $E$ is unitary in $S$;

(ii) $E$ is left unitary in $S$;

(iii) if $e, a \in S$ are such that $e a=e=e^{2}$ then $a=a^{2}$;

(iv) $E$ is a $\sigma$-class of $S$;

(v) $\mathscr{R} \cap \sigma=l_{s}$.

An inverse semigroup $S$ whose semilattice is unitary in $S$ will be called an $E$-unitary inverse semigroup. In what follows we shall use, without comment, some of the other characterisations of such semigroups provided by Lemma 1.6. E-unitary inverse semigroups have been studied by Saitô [20], McAlister [4, 5], O'Carroll [12, 13], Reilly [16] and others. Saitô and McAlister call them "proper inverse semigroups", while O'Carroll refers to them as "reduced inverse semigroups". The term " $E$-unitary" was suggested by A. H. Clifford.

By an $F$-inverse semigroup, McFadden and O'Carroll [7] mean an inverse semigroup $S$, with least group congruence $\sigma$, such that each $\sigma$-class has a greatest element under the natural partial ordering of $S$. It is readily checked that every $F$-inverse semigroup is $E$-unitary. The class of $E$-unitary inverse semigroups also contains that of free inverse semigroups (see \$5).

We now introduce a fourth type of congruence on an inverse semigroup $S$. A congruence $\rho$ on $S$ will be termed $E$-unitary if and only if $S / \rho$ is $E$-unitary. This paper is largely devoted to a study of such congruences.

The next lemma exhibits relationships between some of the concepts discussed above.

LEMMA 1.7. Let $\rho$ be a congruence on an E-unitary inverse semigroup $S$ with semilattice $E$ and least group congruence $\sigma$. The following conditions on $\rho$ are equivalent:

(i) $\rho$ is idempotent-determined;

(ii) $\rho=\pi^{*}$ for some normal equivalence $\pi$ on $E$;

(iii) $\rho \subseteq \sigma$.

Now suppose that $\rho$ satisfies these conditions. Then $\rho$ is E-unitary. Moreover, if $S^{1}$ is an F-inverse semigroup then so also is $(S / \rho)^{1}$.

Proof. (i) implies (ii). Let $\rho$ be idempotent-determined. Write $\pi=\rho \cap(E \times E)$. Then $\pi$ is a normal equivalence on $E$ and $\pi^{*} \subseteq \rho$. We show that $\rho=\pi^{*}$. By (1.2) it suffices to show that, for all $e \in E, e \rho \subseteq e \pi^{*}$. Let $e \in E$ and let $a \in e \rho$. Then $a \in E$, since $\rho$ is idempotentdetermined, and so $(a, e) \in \rho \cap(E \times E)=\pi \subseteq \pi^{*}$. Thus $a \in e \pi^{*}$, which shows that $e \rho \subseteq e \pi^{*}$. It follows that $\rho=\pi^{*}$.

(ii) implies (iii). Let $\rho=\pi^{*}$, where $\pi$ is a normal equivalence on $E$. Since $E \times E \subseteq \sigma$ we have that $(\rho \cap \sigma) \cap(E \times E)=\rho \cap(E \times E)=\pi$ and so, since $\rho=\pi^{*}, \rho \subseteq \rho \cap \sigma$. Hence $\rho=\rho \cap \sigma$; that is, $\rho \subseteq \sigma$.

(iii) implies (i). Let $\rho \subseteq \sigma$. Suppose that $(a, e) \in \rho$, where $e \in E$. Then $(a, e) \in \sigma$ and so, by (1.4), there exists $f \in E$ such that $f a=f e$. Hence $(e f)=(e f) a$. Therefore, since $S$ is $E$-unitary, $a \in E$. Thus $\rho$ is idempotent-determined.

It follows that (i), (ii), (iii) are equivalent. (The hypothesis that $S$ is $E$-unitary was used only to show that (iii) implies (i).)

Now suppose that $\rho$ satisfies (i), (ii) and (iii). We show that $S / \rho$ is $E$-unitary. Let 
$f, b \in S / \rho$ be such that $f^{2}=f$ and $(f b)^{2}=f b$. By (1.1), there exist $e, g \in E$ such that $e \rho=f$ and $g \rho=f b$. Let $a \in S$ be such that $a \rho=b$. Then $(e a, g) \in \rho$. But $\rho$ is idempotent-determined. Hence $e a \in E$ and so $a \in E$, since $S$ is $E$-unitary. Thus $b^{2}=b$. Therefore $S / \rho$ is $E$-unitary.

Finally, suppose also that $S^{1}$ is an $F$-inverse semigroup. We may assume that $S \neq E$. Let $\sigma^{1}, \sigma_{1}, \sigma_{1}^{1}$ denote the least group congruences on $S^{1}, S / \rho,(S / \rho)^{1}$ respectively. Since $S / \rho$ is $E$-unitary, the $\sigma_{1}^{1}$-class of $(S / \rho)^{1}$ containing the idempotents of $S / \rho$ consists entirely of idempotents and so has a greatest element, namely the identity. Now let $a \in S \backslash E$. We show that the $\sigma_{1}^{1}$-class of $(S / \rho)^{1}$ containing a $\rho$ has a greatest element. First we note that this class coincides with the $\sigma_{1}$-class of $S / \rho$ containing $a \rho$; for $a \rho$ contains no idempotent of $S$ and so, by (1.1), cannot be an idempotent of $S / \rho$. But $S^{1}$ is an $F$-inverse semigroup. Hence, since $a \notin E, a \sigma^{1}=a \sigma$ and so there exists $m \in S$ such that (i) $(a, m) \in \sigma$ and (ii) $b \leqslant m$ for all $b \in S$ such that $(a, b) \in \sigma$. But $\rho \subseteq \sigma$ and so, from $(1.5), \sigma_{1}=\sigma / \rho$. Hence $(a \rho, m \rho) \in \sigma_{1}$; also, for all $b \in S$ such that $(a \rho, b \rho) \in \sigma_{1}$, we have that $b \leqslant m$ and therefore that $b \rho \leqslant m \rho$. This shows that the $\sigma_{1}$-class of $S / \rho$ containing $a \rho$ has a greatest element, namely $m \rho$. Thus $(S / \rho)^{1}$ is an $F$-inverse semigroup.

2. P-semigroups. In studying $E$-unitary inverse semigroups, McAlister [4] introduced the important concept of a $P$-semigroup. For completeness, we start this section by recalling the definition of such a semigroup and some of its properties.

By a $M c$ Alister triple we shall mean a triple $(G, \mathscr{X}, \mathscr{Y})$ consisting of a group $G$, a partially ordered set $(\mathscr{X}, \leqslant)$ and a nonempty subset $\mathscr{Y}$ of $\mathscr{X}$ satisfying the following conditions:

(T1) $\mathscr{X}$ is lower directed;

(T2) $\mathscr{Y}$ is an ideal of $\mathscr{X}$ and a lower semilattice under $\leqslant$;

(T3) there exists an action $(g, A) \mapsto g A$ of $G$ on $\mathscr{X}$ by order automorphisms;

(T4) $\mathscr{X}=G \mathscr{Y}$.

It can be shown that if $(G, \mathscr{X}, \mathscr{Y})$ is a McAlister triple then it also satisfies a further condition:

(T5) for all $g \in G$ there exists $A \in \mathscr{Y}$ such that $g A \in \mathscr{Y}$.

Conversely, any triple $(G, \mathscr{X}, \mathscr{Y})$ consisting of a group $G$, a partially ordered set $\mathscr{X}$ and a nonempty subset $\mathscr{Y}$ of $\mathscr{X}$ satisfying (T2), (T3), (T4) and (T5) must necessarily satisfy (T1) and is therefore a McAlister triple.

Let $(G, \mathscr{X}, \mathscr{Y})$ be a McAlister triple. The greatest lower bound of $A, B \in \mathscr{X}$, if such an element exists, will be denoted by $A \wedge B$. From (T2) it follows that, for all $A, B \in \mathscr{Y}, A \wedge B$ exists. Accordingly, we call $\mathscr{Y}$ a "lower subsemilattice" of $\mathscr{X}$. It is a consequence of (T3) that if $A$ and $B$ are elements of $\mathscr{X}$ such that $A \wedge B$ exists then, for any $g \in G, g A \wedge g B$ exists and is equal to $g(A \wedge B)$.

Now let us write

$$
S=\left\{(A, g) \in \mathscr{Y} \times G: g^{-1} A \in \mathscr{Y}\right\} .
$$

It is straightforward to verify that if $(A, g),(B, h) \in S$ then (i) $A \wedge g B$ exists and lies in $\mathscr{Y}$, (ii) $(g h)^{-1}(A \wedge g B) \in \mathscr{Y}$. Thus we can define a multiplication in $S$ by the rule that

$$
(A, g)(B, h)=(A \wedge g B, g h)
$$

Under this multiplication, $S$ is an $E$-unitary inverse semigroup [4, $\$ 1]$. We denote $S$ by $P(G, \mathscr{X}, \mathscr{Y})$. Any semigroup constructed in this way is called a $P$-semigroup. 
Some elementary and easily established facts about $S=P(G, \mathscr{X}, \mathscr{Y})$ should be noted. Let $E$ denote the semilattice of $S$ and let 1 denote the identity of $G$. Then $E=\{(A, 1)$ : $A \in \mathscr{Y}\}$ and this is order isomorphic to $\mathscr{Y}$. Also, $(A, g)^{-1}=\left(g^{-1} A, g^{-1}\right)$ for all $(A, g) \in S$. Green's equivalences are readily characterised; in particular,

$$
((A, g),(B, h)) \in \mathscr{H} \Leftrightarrow A=B \text { and } g^{-1} A=h^{-1} B .
$$

Now let $\sigma$ denote the least group congruence on $S$. Then

$$
((A, g),(B, h)) \in \sigma \Leftrightarrow g=h .
$$

Moreover, the natural partial ordering of $S$ is given by

$$
(A, g) \leqslant(B, h) \Leftrightarrow A \leqslant B \text { and } g=h .
$$

The importance of $P$-semigroups stems from the following theorems due to McAlister [5, Theorem 2.6; 4, Corollary 2.5].

(A) Every $E$-unitary inverse semigroup is isomorphic to a $P$-semigroup.

(B) Every inverse semigroup is isomorphic to the quotient of a $P$-semigroup by an idempotent-separating congruence.

In $\$ 5$ we shall obtain proofs of both theorems by applying our results on $E$-unitary congruences to the case of free inverse semigroups.

McAlister [5, Theorem 2.8] has also shown that $S=P(G, \mathscr{X}, \mathscr{Y})$ is an $F$-inverse semigroup if and only if $\mathscr{X}$ is a lower semilattice and $\mathscr{Y}$ has a greatest element.

To conclude our survey of basic properties of $P$-semigroups we restate the isomorphism theorem [5, Theorem 1.3]. Let $(G, \mathscr{X}, \mathscr{Y}),\left(G^{\prime}, \mathscr{X}^{\prime}, \mathscr{Y}^{\prime}\right)$ be McAlister triples and let $S, S^{\prime}$ denote the corresponding $P$-semigroups. Then $S \cong S^{\prime}$ if and only if there exists an isomorphism $\theta: G \rightarrow G^{\prime}$ and an order isomorphism $\phi: \mathscr{X} \rightarrow \mathscr{X}^{\prime}$ such that (i) $\mathscr{Y} \phi=\mathscr{Y}^{\prime}$ and (ii) $(g A) \phi=$ $(g \theta)(A \phi)$ for all $g \in G$ and all $A \in \mathscr{X}$.

The remainder of this section is devoted to an analysis of certain types of congruences on $P$-semigroups. This will be continued in $\$ 3$ and $\$ 4$.

We start with a definition. Let $(G, \mathscr{X}, \mathscr{Y})$ be a McAlister triple. By a $T$-congruence on $\mathscr{X}$ we shall mean an equivalence $\kappa$ on $\mathscr{X}$ satisfying the following conditions:

(Cl) if $A, C, D \in \mathscr{X}$ are such that $A \leqslant C$ and $(C, D) \in \kappa$ then there exists $B \in \mathscr{X}$ such that $B \leqslant D$ and $(A, B) \in \kappa$;

(C2) each $\kappa$-class is convex;

(C3) $k \cap(\mathscr{Y} \times \mathscr{Y})$ is a congruence on $\mathscr{Y}$;

(C4) if $A, B \in \mathscr{X}$ are such that $(A, B) \in \kappa$ then, for all $g \in G,(g A, g B) \in \kappa$.

Let $\kappa$ be a $T$-congruence on $\mathscr{X}$. We define a relation $\leqslant$ on $\mathscr{X} / \kappa$ by the rule that, for $\mathscr{A}, \mathscr{B} \in \mathscr{X} / \kappa$,

$$
\mathscr{A} \leqslant \mathscr{B} \Leftrightarrow A \leqslant B \text { for some } A \in \mathscr{A}, B \in \mathscr{B} \text {. }
$$

From $(\mathrm{Cl})$ it follows that

$$
\mathscr{A} \leqslant \mathscr{B} \Leftrightarrow \text { for all } B \in \mathscr{B} \text { there exists } A \in \mathscr{A} \text { such that } A \leqslant B .
$$


The relation $\leqslant$ on $\mathscr{X} / \kappa$ is clearly reflexive. From (2.5) and (C2) we see that it is antisymmetric and from (2.5) we see that it is transitive. Thus $\leqslant$ is a partial ordering of $\mathscr{X} / \kappa$. We now define a subset $\mathscr{Y} / \kappa$ of $\mathscr{X} / \kappa$ by writing

$$
\mathscr{Y} / \kappa=\{A \kappa: A \in \mathscr{Y}\}
$$

Also, by (C4), we can define a mapping from $G \times \mathscr{X} / \kappa$ to $\mathscr{X} / \kappa$ by the rule that

$$
g(A \kappa)=(g A) \kappa \quad(g \in G, A \in \mathscr{X}) .
$$

It follows, again from (C4), that if $A, B \in \mathscr{X}$ are such that $A \kappa \leqslant B \kappa$ then, for all $g \in G$, $g(A \kappa) \leqslant g(B \kappa)$. From this we see easily that the mapping defined by (2.7) is an action of $G$ on $\mathscr{X} / \kappa$ by order automorphisms.

As in [9] we say that an inverse subsemigroup $U$ of an inverse semigroup $T$ is full if and only if $U$ contains all the idempotents of $T$. Then we have

Lemma 2.8. Let $(G, \mathscr{X}, \mathscr{Y})$ be a McAlister triple and let $\kappa$ be a T-congruence on $\mathscr{X}$. Let $\mathscr{X} / \kappa$ be partially ordered by (2.4), let $\mathscr{Y} / \kappa$ be defined by (2.6) and let $G$ act on $\mathscr{X} / \kappa$ according to (2.7). Then $(G, \mathscr{X} / \kappa, \mathscr{Y} / \kappa)$ is a McAlister triple.

Write $S=P(G, \mathscr{X}, \mathscr{Y}), T=P(G, X / \kappa, \mathscr{Y} / \kappa)$ and let $\theta: S \rightarrow T$ be defined by $(A, g) \theta=$ $(A \kappa, g)$. Then

(i) $\theta$ is a homomorphism and $S \theta$ is a full inverse subsemigroup of $T$;

(ii) the congruence $\theta \circ \theta^{-1}$ on $S$ is idempotent-determined;

(iii) if each $\kappa$-class of $\mathscr{X}$ is lower directed then $\theta$ is surjective;

(iv) if each $\kappa$-class of $\mathscr{X}$ is lower directed and $\mathscr{X}$ is a lower semilattice then $\mathscr{X} / \kappa$ is a lower semilattice.

Proof. We first verify that $(G, \mathscr{X} / \kappa, \mathscr{Y} / \kappa)$ satisfies conditions (T1), (T2), (T3), (T4).

Since $\mathscr{X}$ is lower directed, so also is $\mathscr{X} / \kappa$; thus (T1) holds.

Now consider the subset $\mathscr{Y} / \kappa$ of $\mathscr{X} / \kappa$. Let $\mathscr{A} \in \mathscr{X} / \kappa, \mathscr{B} \in \mathscr{Y} / \kappa$ and suppose that $\mathscr{A} \leqslant \mathscr{B}$. Let $B \in \mathscr{B} \cap \mathscr{Y}$. Then, by (2.5), there exists $A \in \mathscr{A}$ such that $A \leqslant B$. Since $\mathscr{O}$ is an ideal of $\mathscr{X}, A \in \mathscr{Y}$ and so $\mathscr{A} \in \mathscr{Y} / \kappa$. Thus $\mathscr{Y} / \kappa$ is an ideal of $\mathscr{X} / \kappa$. We now show that $\mathscr{Y} / \kappa$ is a lower subsemilattice of $\mathscr{X} / \kappa$. It will be enough to show that

$$
(C \wedge D) \kappa=C \kappa \wedge D \kappa \quad(C, D \in \mathscr{Y}) .
$$

Let $C, D \in \mathscr{Y}$. First, we note that $(C \wedge D) \kappa \leqslant C \kappa, D \kappa$. Now suppose that $\mathscr{M} \in \mathscr{X} / \kappa$ is such that $\mathscr{U} \leqslant C \kappa, D_{\kappa}$. We prove that $\mathscr{M} \leqslant(C \wedge D)_{\kappa}$. By (2.5), there exist $M_{1}, M_{2} \in \mathscr{M}$ such that $M_{1} \leqslant C$ and $M_{2} \leqslant D$. Since $\mathscr{Y}$ is an ideal of $\mathscr{X}, M_{1}$ and $M_{2}$ lie in $\mathscr{Y}$. Hence $M_{1} \wedge M_{2} \leqslant C \wedge D$. But $\left(M_{1}, M_{2}\right) \in \kappa$ and so, by (C3), $\left(M_{1} \wedge M_{2}, M_{2}\right) \in \kappa$. Hence $\mathscr{H}=M_{2} \kappa=\left(M_{1} \wedge M_{2}\right) \kappa \leqslant$ $(C \wedge D) \kappa$. Thus $(\alpha)$ holds. This shows that $\mathscr{Y} / \kappa$ is a lower subsemilattice of $\mathscr{X} / \kappa$. Hence (T2) holds.

Equation (2.7) defines an action of $G$ on $\mathscr{X} / \kappa$ by order automorphisms, as already noted; thus (T3) holds. Finally, we show that $\mathscr{X} / \kappa=G(\mathscr{Y} / \kappa)$. Let $A \in \mathscr{X}$. Then there exist $B \in \mathscr{Y}$ and $g \in G$ such that $A=g B$. Hence $A \kappa=(g B) \kappa=g(B \kappa)$. Thus (T4) holds. Consequently, $(G, \mathscr{X} / \kappa, \mathscr{Y} / \kappa)$ is a McAlister triple. 
Now let $S=P(G, \mathscr{X}, \mathscr{Y}), T=P(G, \mathscr{X} / \kappa, \mathscr{Y} / \kappa)$. Let $(A, g) \in S$. Then $g^{-1} A \in \mathscr{Y}$ and so $g^{-1}(A \kappa)=\left(g^{-1} A\right) \kappa \in \mathscr{Y} / \kappa$. This enables us to define $\theta: S \rightarrow T$ by the rule that $(A, g) \theta=(A \kappa, g)$.

(i) Let $(A, g),(B, h) \in S$. To show that $\theta$ is a homomorphism it suffices to prove that $A \kappa \wedge g(B \kappa)=(A \wedge g B) \kappa$. Now

$$
\begin{aligned}
A \kappa \wedge g(B \kappa) & =g\left[g^{-1}(A \kappa) \wedge B \kappa\right] \\
& =g\left[\left(g^{-1} A\right) \kappa \wedge B \kappa\right] \\
& =g\left[\left(g^{-1} A \wedge B\right) \kappa\right], \text { by }(\alpha), \\
& =\left[g\left(g^{-1} A \wedge B\right)\right] \kappa=(A \wedge g B)_{\kappa},
\end{aligned}
$$

as required. Since the idempotents of $T$ are the elements of the form $(A \kappa, 1)=(A, 1) \theta$ $(A \in \mathscr{Y})$, where 1 denotes the identity of $G, S \theta$ is a full inverse subsemigroup of $T$.

(ii) Let $(A, g),(B, h) \in S$ be such that $(A, g) \theta=(B, h) \theta$. Then $g=h$ and so, by (2.2), $\theta \circ \theta^{-1} \subseteq \sigma$, the least group congruence on $S$. Hence, since $S$ is $E$-unitary, $\theta \circ \theta^{-1}$ is idempotentdetermined, by Lemma 1.7 .

(iii) Suppose that each $\kappa$-class of $\mathscr{X}$ is lower directed. Let $(A \kappa, g) \in T$, where $A \in \mathscr{Y}, g \in G$. Then $g^{-1}(A \kappa) \in \mathscr{Y} / \kappa$; that is, $\left(g^{-1} A\right) \kappa \in \mathscr{Y} / \kappa$. Hence there exists $B \in \mathscr{Y}$ such that $\left(g^{-1} A, B\right) \in \kappa$. Since each $\kappa$-class is lower directed, there exists $C \in \mathscr{X}$ such that $C \leqslant g^{-1} A, C \leqslant B$ and $\left(C, g^{-1} A\right) \in \kappa$. Now $g C \leqslant A$, since $C \leqslant g^{-1} A$; hence $g C \in \mathscr{Y}$, since $A \in \mathscr{Y}$. Also $C \in \mathscr{Y}$, since $B \in \mathscr{Y}$. Hence $(g C, g) \in S$. Furthermore, $(g C, A)=\left(g C, g\left(g^{-1} A\right)\right) \in \kappa$, by (C4). Thus $(g C) \kappa=A \kappa$ and so $(g C, g) \theta=(A \kappa, g)$. Hence $\theta$ is surjective.

(iv) Suppose that each $\kappa$-class of $\mathscr{X}$ is lower directed and that $\mathscr{X}$ is a lower semilattice. Let $A, B \in \mathscr{X}$. To show that $\mathscr{X} / \kappa$ is a lower semilattice it is enough to prove that

$$
(A \wedge B) \kappa=A \kappa \wedge B \kappa .
$$

Clearly, $(A \wedge B) \kappa \leqslant A \kappa, B \kappa$. Let $\mathscr{M} \in \mathscr{X} / \kappa$ be such that $\mathscr{M} \leqslant A \kappa, B \kappa$. By $(2.5)$, there exist $M_{1}, M_{2} \in \mathscr{M}$ such that $M_{1} \leqslant A$ and $M_{2} \leqslant B$. Hence, since $\mathscr{M}$ is lower directed, there exists $M_{3} \in \mathscr{M}$ such that $M_{3} \leqslant M_{1}$ and $M_{3} \leqslant M_{2}$. It follows that $M_{3} \leqslant A \wedge B$ and so $\mathscr{M} \leqslant(A \wedge B) \kappa$. Thus $A \kappa \wedge B \kappa$ exists and is equal to $(A \wedge B) \kappa$.

Henceforth if $(G, \mathscr{X}, \mathscr{Y})$ is a McAlister triple and $\kappa$ is a $T$-congruence on $\mathscr{X}$ then $(G, \mathscr{X} / \kappa, \mathscr{Y} / \kappa)$ will denote the McAlister triple formed in the manner described above.

We now describe a second method for forming new McAlister triples from a given one. By the kernel of a McAlister triple $(G, \mathscr{X}, \mathscr{Y})$ we shall mean the set $\{g \in G: g A=A$ for all $A \in \mathscr{X}\}$. It will be denoted by $\operatorname{ker}(G, \mathscr{X}, \mathscr{Y})$. Evidently $\operatorname{ker}(G, \mathscr{X}, \mathscr{Y})$ is a normal subgroup of $G$.

The proof of the following result is straightforward and will be omitted.

LEMMA 2.9. Let $(G, \mathscr{X}, \mathscr{Y})$ be a McAlister triple and let $N$ be a normal subgroup of $G$ such that $N \subseteq \operatorname{ker}(G, \mathscr{X}, \mathscr{Y})$. Then the mapping from $G / N \times \mathscr{X}$ to $\mathscr{X}$, defined by the rule that

$$
(g N) A=g A \quad(g \in G, A \in \mathscr{X}),
$$

is an action of $G / N$ on $\mathscr{X}$ by order automorphisms and, with respect to this action, $(G / N, \mathscr{X}, \mathscr{Y})$ is a McAlister triple. 

Then

Write $S=P(G, \mathscr{X}, \mathscr{Y}), T=P(G / N, \mathscr{X}, \mathscr{Y})$ and let $\theta: S \rightarrow T$ be defined by $(A, g) \theta=(A, g N)$.

(i) $\theta$ is a surjective homomorphism,

(ii) the congruence $\theta \circ \theta^{-1}$ on $S$ is idempotent-separating.

We remark that, in general, not every idempotent-separating congruence on $S$ is of the form $\theta \circ \theta^{-1}$ for some $\theta$ defined as above.

For the remainder of the paper, if $(G, \mathscr{X}, \mathscr{Y})$ is a McAlister triple and if $N$ is a normal subgroup contained in its kernel then $(G / N, \mathscr{X}, \mathscr{Y})$ will denote the McAlister triple defined in Lemma 2.9.

3. Idempotent-determined congruences on P-semigroups. We now apply the foregoing results to the problem of describing the quotient of a $P$-semigroup $S$ by an idempotent-determined congruence. Since $S$ is $E$-unitary, it follows from Lemma 1.7 that a congruence $\rho$ on $S$ is idempotent-determined if and only if $\rho=\pi^{*}$ for some normal equivalence $\pi$ on the semilattice of $S$. Thus we shall examine quotients of the form $S / \pi^{*}$.

First we have a technical lemma.

Lemma 3.1. Let $(G, \mathscr{X}, \mathscr{Y})$ be a McAlister triple, let $S=P(G, \mathscr{X}, \mathscr{Y})$ and let $\hat{\pi}$ be $a$ congruence on $\mathscr{Y}$. Let $C, D \in \mathscr{X}$ have the property that, for some $n$, there exist elements $C_{0}=C, C_{1}, C_{2}, \ldots, C_{n}=D$ in $\mathscr{X}$ and $g_{1}, g_{2}, \ldots, g_{n} \in G$ such that $g_{i} C_{i-1}, g_{i} C_{i} \in \mathscr{Y}$ and $\left(g_{i} C_{i-1}, g_{i} C_{i}\right) \in \hat{\pi}(i=1, \ldots, n)$. Let $A \in \mathscr{X}$ be such that $A \leqslant C$. Then

$$
A \wedge C_{1} \wedge C_{2} \wedge \ldots \wedge C_{i}
$$

exists $(i=1, \ldots, n)$. Write $M_{0}=A, M_{i}=A \wedge C_{1} \wedge \ldots \wedge C_{i}$. Then $g_{i} M_{i-1}, g_{i} M_{i} \in \mathscr{Y}$ and $\left(g_{i} M_{i-1}, g_{i} M_{i}\right) \in \hat{\imath}(i=1, \ldots, n)$.

Proof. Let $1 \leqslant i \leqslant n$ and suppose that we have shown that $M_{i-1}$ exists. Then $M_{i-1} \leqslant$ $C_{i-1}$ and so $g_{i} M_{i-1} \leqslant g_{i} C_{i-1}$. But $g_{i} C_{i-1} \in \mathscr{Y}$; hence $g_{i} M_{i-1} \in \mathscr{Y}$. Also $g_{i} C_{i} \in \mathscr{Y}$. Thus $g_{i} M_{i-1} \wedge g_{i} C_{i}$ exists and lies in $\mathscr{Y}$. It follows that $M_{i}\left(=M_{i-1} \wedge C_{i}\right)$ exists and that $g_{i} M_{i} \in \mathscr{Y}$. Now $\left(g_{i} C_{i-1}, g_{i} C_{i}\right) \in \hat{\pi}$ and so $\left(g_{i} M_{i-1} \wedge g_{i} C_{i-1}, g_{i} M_{i-1} \wedge g_{i} C_{i}\right) \in \hat{\pi}$, since $\hat{t}$ is a congruence on $\mathscr{Y}$. Hence $\left(g_{i} M_{i-1}, g_{i} M_{i}\right) \in \hat{\pi}$. But $M_{i-1}$ exists for $i=1$. Hence, taking $i=1,2, \ldots, n$ successively, we obtain the result.

THEOREM 3.2. Let $(G, \mathscr{X}, \mathscr{Y})$ be a McAlister triple, let $S=P(G, \mathscr{X}, \mathscr{Y})$, let $E$ denote the semilattice of $S$ and let 1 denote the identity of $G$. Let $\pi$ be a normal equivalence on $E$. Then the relation $\hat{A}$ defined on $\mathscr{Y}$ by

$$
(A, B) \in \hat{\pi} \Leftrightarrow((A, 1),(B, 1)) \in \pi
$$

is a congruence on $\mathscr{Y}$. Let $\kappa$ denote the transitive closure of the following relation on $X$ :

$$
\{(C, D): g C, g D \in \mathscr{Y} \text { and }(g C, g D) \in \hat{\pi} \text { for some } g \in G\} \text {. }
$$

Then $\kappa$ is a T-congruence on $\mathscr{X}$ and $S / \pi^{*} \cong P(G, \mathscr{X} / \kappa, \mathscr{Y} / \kappa)$. Moreover, if $\mathscr{X}$ is a lower semilattice then so also is $\mathscr{X} / \kappa$. 
Proof. Since $A \mapsto(A, 1)$ is an isomorphism from $\mathscr{Y}$ to $E$, and $\pi$ is a normal equivalence on $E, \hat{\pi}$ is a congruence on $\mathscr{Y}$. By (T4), $\kappa$ is reflexive. Since $\kappa$ is evidently symmetric and transitive, it is an equivalence on $\mathscr{X}$. We now show that it is a $T$-congruence.

Let $A, C, D \in \mathscr{X}$ be such that $A \leqslant C$ and $(C, D) \in \kappa$. Since $(C, D) \in \kappa$ and $\hat{\pi}$ is a congruence on $\mathscr{Y}$ there exist elements $C_{i}, g_{i}$ satisfying the hypotheses of Lemma 3.1. Thus, by the lemma, $M_{i}=A \wedge C_{0} \wedge C_{1} \wedge \ldots \wedge C_{i}$ exists $(i=0, \ldots, n)$; further, $g_{i} M_{i-1}, g_{i} M_{i} \in \mathscr{Y}$ and $\left(g_{i} M_{i-1}, g_{i} M_{i}\right) \in \hat{\pi}(i=1, \ldots, n)$. Write $B=C_{0} \wedge C_{i} \wedge \ldots \wedge C_{n}$. Then

$$
B \leqslant C, D ; \quad(A, A \wedge B) \in \kappa .
$$

Since $A \wedge B \leqslant D$ it follows that $\kappa$ satisfies $(\mathrm{Cl})$.

Next we show that each $\kappa$-class is convex. Let $A, C, D \in \mathscr{X}$ be such that $D \leqslant A \leqslant C$ and $(C, D) \in \kappa$. From $(\alpha)$, there exists $B \in \mathscr{X}$ such that $B \leqslant D$ and $(A, A \wedge B) \in \kappa$. Since $B \leqslant D \leqslant A$ we have that $A \wedge B=B$. Hence $(A, B) \in \kappa$. On the other hand, $(\alpha)$ holds with $A=C$. Hence $(C, C \wedge B) \in \kappa$ and so, since $B \leqslant C,(C, B) \in \kappa$. Combining these results, we see that $(A, C) \in \kappa$. This shows that $C \kappa$ is convex. Thus (C2) is satisfied.

We now prove that $\kappa \cap(\mathscr{Y} \times \mathscr{Y})=\hat{\pi}$. Clearly $\hat{\pi} \subseteq \kappa \cap(\mathscr{Y} \times \mathscr{Y})$. Accordingly, let $(C, D) \in$ $\kappa \cap(\mathscr{Y} \times \mathscr{Y})$. Then there exist elements $C_{i}, g_{i}$ satisfying the hypotheses of Lemma 3.1. Taking $A=C$ in the lemma, we see that $C_{0} \wedge C_{1} \wedge \ldots \wedge C_{i}=M_{i}$ exists for $i=0, \ldots, n$; furthermore, $g_{i} M_{i-1}, g_{i} M_{i} \in \mathscr{Y}$ and $\left(g_{i} M_{i-1}, g_{i} M_{i}\right) \in \hat{\pi} \quad(i=1, \ldots, n)$. Now since $M_{i} \leqslant C_{0}=C \in \mathscr{Y}$ it follows that $M_{i} \in \mathscr{Y}(i=0, \ldots, n)$. Hence $\left(M_{i-1}, g_{i}^{-1}\right) \in S(i=1, \ldots, n)$ and a simple computation shows that

$$
\begin{aligned}
\left(M_{i-1}, g_{i}^{-1}\right)\left(g_{i} M_{i-1}, 1\right)\left(M_{i-1}, g_{i}^{-1}\right)^{-1} & =\left(M_{i-1}, 1\right), \\
\left(M_{i-1}, g_{i}^{-1}\right)\left(g_{i} M_{i}, 1\right)\left(M_{i-1}, g_{i}^{-1}\right)^{-1} & =\left(M_{i}, 1\right) .
\end{aligned}
$$

Thus since $\pi$ is a normal equivalence on $E$ and $\left(\left(g_{i} M_{i-1}, 1\right),\left(g_{i} M_{i}, 1\right)\right) \in \pi$, it follows that $\left(\left(M_{i-1}, 1\right),\left(M_{i}, 1\right)\right) \in \pi$. Hence $\left(M_{i-1}, M_{i}\right) \in \hat{\pi}$. But this holds for $i=1, \ldots, n$. Hence $\left(M_{0}, M_{n}\right) \in \hat{\pi}$; that is, $\left(C, C_{0} \wedge C_{1} \wedge \ldots \wedge C_{n}\right) \in \hat{\pi}$. Since $C_{n}=D$ a similar argument will prove that $\left(D, C_{0} \wedge C_{1} \wedge \ldots \wedge C_{n}\right) \in \hat{\pi}$. Hence $(C, D) \in \hat{\pi}$. Thus we have shown that $\kappa \cap(\mathscr{Y} \times \mathscr{Y}) \subseteq \hat{\pi}$. Consequently, $\kappa \cap(\mathscr{Y} \times \mathscr{Y})=\hat{\pi}$. Since $\hat{\pi}$ is a congruence on $\mathscr{Y}, \kappa$ satisfies (C3).

Last, we show that $\kappa$ satisfies (C4). Let $(C, D) \in \kappa$ and let $h \in G$. Then there exist elements $C_{0}=C, C_{1}, \ldots, C_{n}=D$ in $\mathscr{X}$ and $g_{1}, \ldots, g_{n} \in G$ such that $g_{i} C_{i-1}, g_{i} C_{i} \in \mathscr{Y}$ and $\left(g_{i} C_{i-1}, g_{i} C_{i}\right) \in \hat{\pi}(i=1, \ldots, n)$. Write $C_{i}{ }^{\prime}=h C_{i}$ and $g_{i}{ }^{\prime}=g_{i} h^{-1}$. Then $C_{0}{ }^{\prime}=h C, C_{n}{ }^{\prime}=h D$. Also $g_{i}{ }^{\prime} C_{i-1}{ }^{\prime}=g_{i} C_{i-1} \in \mathscr{Y}, g_{i}{ }^{\prime} C_{i}{ }^{\prime}=g_{i} C_{i} \in \mathscr{Y}$ and $\left(g_{i}{ }^{\prime} C_{i}{ }^{\prime}-1, g_{i}{ }^{\prime} C_{i}{ }^{\prime}\right) \in \hat{\pi}(i=1, \ldots, n)$. Hence $(h C, h D) \in \kappa$. Thus $\kappa$ satisfies $(C 4)$. Hence $\kappa$ is a $T$-congruence on $\mathscr{X}$.

We note next that every $\kappa$-class of $\mathscr{X}$ is lower directed. To see this, let $(C, D) \in \kappa$. Then there exists $B \in \mathscr{X}$ such that $(\alpha)$ holds for any $A \in \mathscr{X}$ such that $A \leqslant C$. Take $A=C$. Then $B \leqslant C, D$ and $(C, B) \in \kappa$.

Now let us apply Lemma 2.8. First, $(G, \mathscr{X} / \kappa, \mathscr{Y} / \kappa)$ is a McAlister triple. Let $S=$ $P(G, \mathscr{X}, \mathscr{Y}), T=P(G, \mathscr{X} / \kappa, \mathscr{Y} / \kappa)$ and define $\theta: S \rightarrow T$ by $(A, g) \theta=(A \kappa, g)$. Then $\theta$ is a homomorphism. Also, $\theta$ is surjective, since each $\kappa$-class is lower directed. Thus $S / \theta \circ \theta^{-1} \cong T$. Moreover, $\theta \circ \theta^{-1}$ is idempotent-determined. Hence, by Lemma 1.7, since $S$ is $E$-unitary, $\theta \circ \theta^{-1}=\pi_{1}^{*}$ for some normal equivalence $\pi_{1}$ on $E$. Now, for all $A, B \in \mathscr{Y}$, 


$$
\begin{aligned}
& ((A, 1),(B, 1)) \in \pi_{1} \\
\Leftrightarrow & (A, 1) \theta=(B, 1) \theta, \text { since } \theta \circ \theta^{-1} \cap(E \times E)=\pi_{1}, \\
\Leftrightarrow & (A, B) \in \kappa, \\
\Leftrightarrow & (A, B) \in \hat{\pi}, \text { since } \kappa \cap(\mathscr{Y} \times \mathscr{Y})=\hat{\pi}, \\
\Leftrightarrow & ((A, 1),(B, 1)) \in \pi .
\end{aligned}
$$

Hence $\pi_{1}=\pi$. Consequently, $S / \pi^{*} \cong T$, as required.

Finally, since each $\kappa$-class of $\mathscr{X}$ is lower directed it follows from Lemma 2.8 (iv) that if $\mathscr{X}$ is a lower semilattice then so also is $\mathscr{X} / \kappa$.

4. Idempotent-separating congruences on P-semigroups. Let $(G, \mathscr{X}, \mathscr{Y})$ be a McAlister triple, let $S=P(G, \mathscr{X}, \mathscr{Y})$ and let $\rho$ be an idempotent-separating congruence on $S$. Since $\rho \subseteq \mathscr{H}$, it follows from (2.1) that if $(A, g),(B, h) \in S$ are $\rho$-equivalent then $A=B$. Denote the identity of $G$ by 1 . We shall be interested in the following two subsets of $G$ associated with $\rho$ :

$M_{\rho}=\{g \in G:((A, g),(A, 1)) \in \rho$ for all $A \in \mathscr{Y}$ for which $(A, g) \in S\}$

$N_{p}=\{g \in G:((A, g),(A, 1)) \in \rho$ for some $A \in \mathscr{Y}$ for which $(A, g) \in S\}$.

Clearly, $l \in M_{\rho}, N_{\rho}$. Also $M_{\rho} \subseteq N_{\rho}$. Furthermore, if $\xi, \eta$ are idempotent-separating congruences on $S$ such that $\xi \subseteq \eta$ then $M_{\xi} \subseteq M_{\eta}$ and $N_{\xi} \subseteq N_{\eta}$.

In the next four lemmas we obtain some further properties of these subsets. To save repetition, we shall assume here that $S=P(G, \mathscr{X}, \mathscr{Y})$ as above.

Lemma 4.1. Let $\rho$ be an idempotent-separating congruence on $S$. Then $N_{\rho}$ is a normal subgroup of $G$.

Proof. Let $g, h \in N_{\rho}$. Then there exist $A, B \in \mathscr{Y}$ such that $(A, g),(B, h) \in S$ and $((A, g),(A, 1)) \in \rho,((B, h),(B, 1)) \in \rho$. Since $\rho$ is a congruence, $\left((A, g)(B, h)^{-1},(A, 1)(B, 1)^{-1}\right) \in \rho$; that is, $\left(\left(A \wedge g h^{-1} B, g h^{-1}\right),(A \wedge B, 1)\right) \in \rho$. But $\rho \subseteq \mathscr{H}$ and so $A \wedge g h^{-1} B=A \wedge B$. Hence $g h^{-1} \in N_{\rho}$ and so $N_{\rho}$ is a subgroup of $G$. The proof that $N_{\rho}$ is normal in $G$ is on similar lines and will be omitted.

By contrast, $M_{\rho}$ need not always be a subsemigroup of $G$. But the situation is simplified when $\rho$ is $E$-unitary, as will now be shown.

LEMMA 4.2. Let $\rho$ be an E-unitary idempotent-separating congruence on $S$. Then $M_{\rho}=N_{\rho}$.

Proof. We need only show that $N_{p} \subseteq M_{p}$. Let $g \in N_{p}$. Then there exists $A \in \mathscr{Y}$ such that $(A, g) \in S$ and $((A, g),(A, 1)) \in \rho$. Let $B$ be any element of $\mathscr{Y}$ such that $(B, g) \in S$. Then $((B, 1)(A, g),(B, 1)(A, 1)) \in \rho$. Hence, $((A \wedge B, 1)(B, g),(A \wedge B, 1)) \in \rho$ and so, since $\rho$ is $E$ unitary, $\left((B, g)^{2},(B, g)\right) \in \rho$. Hence, by $(1.1)$, there exists $C \in \mathscr{Y}$ such that $((B, g),(C, 1)) \in \rho$. But $\rho \subseteq \mathscr{H}$ and so $C=B$. Thus $((B, g),(B, 1)) \in \rho$. This shows that $g \in M_{\rho}$. Hence $N_{\rho} \subseteq M_{p}$. Then

Lемma 4.3. Let $\xi, \eta$ be idempotent-separating congruences on $S$ and let $\eta$ be E-unitary.

$$
\xi \subseteq \eta \Leftrightarrow N_{\xi} \subseteq N_{\eta}
$$


Proof. We have already noted that if $\xi \subseteq \eta$ then $N_{\xi} \subseteq N_{\eta}$. Suppose that $N_{\xi} \subseteq N_{\eta}$. By (1.2), to show that $\xi \subseteq \eta$ it is enough to show that $(A, 1) \xi \subseteq(A, 1) \eta$ for all $A \in \mathscr{Y}$. Let $A \in \mathscr{Y}$ and let $(B, g) \in(A, 1) \xi$. Then, since $\xi \subseteq \mathscr{H}, B=A$. Hence $g \in N_{\xi}$ and so, by hypothesis, $g \in N_{\eta}$. But, since $\eta$ is $E$-unitary, $N_{\eta}=M_{\eta}$, by Lemma 4.2. Hence $g \in M_{\eta}$ and so $(B, g)=$ $(A, g) \in(A, 1) \eta$. Hence $(A, 1) \xi \subseteq(A, 1) \eta$.

It follows from Lemma 4.3 that if $\xi, \eta$ are $E$-unitary idempotent-separating congruences on $S$ then

$$
\xi=\eta \Leftrightarrow N_{\xi}=N_{\eta}
$$

LEMMA 4.4. Let $\rho$ be an idempotent-separating congruence on $S$. Let $N$ be a normal subgroup of $G$ such that $N \subseteq M_{\rho}$. A relation $\kappa$ is defined on $\mathscr{X}$ by the rule that

$$
(A, B) \in \kappa \Leftrightarrow B=n A \text { for some } n \in N \text {. }
$$

Then $\kappa$ is a T-congruence on $\mathscr{X}$ such that $\kappa \cap(\mathscr{Y} \times \mathscr{Y})=t_{\mathscr{Y}}$ and $N \subseteq \operatorname{ker}(G, \mathscr{X} / \kappa, \mathscr{Y} / \kappa)$. Let $T=P(G / N, \mathscr{X} / \kappa, \mathscr{Y} / \kappa)$ and let $\theta: S \rightarrow T$ be defined by $(A, g) \theta=(A \kappa, g N)$. Then

(i) $\theta$ is a surjective homomorphism;

(ii) $\theta \circ \theta^{-1}$ is an E-unitary (idempotent-separating) congruence contained in $\rho$;

(iii) $N_{\theta \circ \theta^{-1}}=N$.

Proof. Since $N$ is a subgroup of $G, \kappa$ is an equivalence on $\mathscr{X}$. We first show that

$$
\kappa \cap(\mathscr{Y} \times \mathscr{Y})=\imath_{\mathscr{y}} .
$$

Let $(A, B) \in \kappa \cap(\mathscr{Y} \times \mathscr{Y})$. We have to show that $A=B$. Since $(A, B) \in \kappa$ there exists $n \in N$ such that $B=n A$. Since $B \in \mathscr{Y},\left(A, n^{-1}\right) \in S$. But $n^{-1} \in N \subseteq M_{\rho}$ and so $\left(\left(A, n^{-1}\right),(A, 1)\right) \in \rho$. Hence $((n A, n),(A, 1))=\left(\left(A, n^{-1}\right)^{-1},(A, 1)^{-1}\right) \in \rho$. But $\rho \subseteq \mathscr{H}$. Hence $n A=A$; that is, $B=A$.

We now verify that $\kappa$ is a $T$-congruence. Let $A, C, D \in \mathscr{X}$ be such that $A \leqslant C$ and $(C, D) \in \kappa$. Then there exists $n \in N$ since that $D=n C$. Hence $n A \leqslant n C=D$; also $(A, n A) \in \kappa$. Thus (C1) is satisfied. It is immediate from $(\alpha)$ that $\kappa$ satisfies (C3). We prove next that it satisfies (C4). Let $(A, B) \in \kappa$ and let $g \in G$. Then $B=n A$ for some $n \in N$ and so $g B=g n A=$ $n^{\prime} g A$, for some $n^{\prime} \in N$, since $N$ is normal in $G$. Thus $(g A, g B) \in \kappa$. Hence $\kappa$ satisfies (C4).

It remains to show that $\kappa$ satisfies (C2). The result will follow if we prove that no two distinct $\kappa$-equivalent elements of $\mathscr{X}$ are comparable. Suppose that there exist $A, B \in \mathscr{X}$ such that $A<B$ and $(A, B) \in \kappa$. We show that this leads to a contradiction. Since $\mathscr{X}=G \mathscr{Y}$, there exists $g \in G$ such that $g B \in \mathscr{Y}$. Now $g A<g B$ and so $g A \in \mathscr{Y}$, since $\mathscr{Y}$ is an ideal of $\mathscr{X}$. But $(g A, g B) \in \kappa$, since $(A, B) \in \kappa$ and $\kappa$ satisfies (C4). Hence $(g A, g B) \in \kappa \cap(\mathscr{Y} \times \mathscr{Y})$ and so, by $(\alpha), g A=g B$. This conflicts with the inequality $g A<g B$ obtained above. Hence each $\kappa$-class is (trivially) convex; that is, $\kappa$ satisfies (C2). We have thus shown that $\kappa$ is a $T$-congruence on $\mathscr{X}$.

By Lemma 2.8, $(G, \mathscr{X} / \kappa, \mathscr{Y} / \kappa)$ is a McAlister triple. Let $n \in N$. Then, for all $A \in \mathscr{X}$, $(n A) \kappa=A \kappa$; that is, $n(A \kappa)=A \kappa$. Thus $N \subseteq \operatorname{ker}(G, \mathscr{X} / \kappa, \mathscr{Y} / \kappa)$. Hence, by Lemma 2.9, we 
can form the McAlister triple $(G / N, \mathscr{X} / \kappa, \mathscr{Y} / \kappa)$, where the action of $G / N$ on $\mathscr{X} / \kappa$ is according to the rule that

$$
(g N)(A \kappa)=g(A \kappa)=(g A) \kappa \quad(g \in G, A \in \mathscr{X}) .
$$

Let $T=P(G / N, \mathscr{X} / \kappa, \mathscr{Y} / \kappa)$ and let $\theta: S \rightarrow T$ be defined by $(A, g) \theta=(A \kappa, g N)$.

(i) Let us denote $P(G, \mathscr{X} / \kappa, \mathscr{Y} / \kappa)$ by $U$. By Lemma 2.8, the mapping $\theta_{1}:(A, g) \mapsto(A \kappa, g)$ is a homomorphism from $S$ to $U$ and, by Lemma 2.9, the mapping $\theta_{2}:(A \kappa, g) \mapsto(A \kappa, g N)$ is a homomorphism from $U$ to $T$. Now $\theta=\theta_{1} \theta_{2}$ and so $\theta$ is a homomorphism. We show further that $\theta$ is surjective. Let $(A \kappa, g N) \in T$, where $A \in \mathscr{Y}, g \in G$. Then $(g N)^{-1}(A \kappa) \in \mathscr{Y} / \kappa$. But $(g N)^{-1}(A \kappa)=\left(g^{-1} N\right)(A \kappa)=g^{-1}(A \kappa)=\left(g^{-1} A\right) \kappa$. Hence there exists $B \in \mathscr{Y}$ such that $\left(B, g^{-1} A\right) \in \kappa$ and so there exists $n \in N$ such that $B=n g^{-1} A$. Since $B \in \mathscr{Y}$ it follows that $\left(A, g n^{-1}\right) \in S$. Moreover, $\left(A, g n^{-1}\right) \theta=(A \kappa, g N)$. Thus $\theta$ is surjective. (It is tempting to assume that $\theta_{1}: S \rightarrow U$ is surjective; but this is not always true.)

(ii) Since $\theta$ is surjective, $S / \theta \circ \theta^{-1} \cong T$. But $T$ is $E$-unitary; hence $\theta \circ \theta^{-1}$ is an $E$-unitary congruence. We now show that $\theta \circ \theta^{-1} \subseteq \rho$. By (1.2), it suffices to show that $(A, 1) \theta \circ \theta^{-1} \subseteq$ $(A, 1) \rho$ for all $A \in \mathscr{Y}$. Let $A \in \mathscr{Y}$ and let $(B, g) \in S$ be such that $(B, g) \theta=(A, 1) \theta$. Then $(A, B) \in \kappa$ and $g N=N$. Since $(A, B) \in \kappa \cap(\mathscr{Y} \times \mathscr{Y})$ it follows from $(\alpha)$ that $A=B$. Thus $(B, g)=(A, g)$. Also, $g \in N \subseteq M_{\rho}$; hence $(A, g) \in(A, 1) \rho$. Thus $(A, 1) \theta \circ \theta^{-1} \subseteq(A, 1) \rho$. Consequently $\theta \circ \theta^{-1} \subseteq \rho$. Since $\rho$ is idempotent-separating, so also is $\theta \circ \theta^{-1}$.

(iii) Let $g \in N_{\theta \circ \theta^{-1}}$. Then there exists $A \in \mathscr{Y}$ such that $(A, g) \in S$ and $(A, g) \theta=(A, 1) \theta$. Thus $g N=N$ and so $g \in N$. Hence $N_{\theta \circ \theta^{-1}} \subseteq N$. Conversely, let $g \in N$. Then, by (T5), there exists $A \in \mathscr{Y}$ such that $g^{-1} A \in \mathscr{Y}$. Thus $(A, g) \in S$ and $(A, g) \theta=(A \kappa, g N)=(A \kappa, N)=(A, 1) \theta$, which shows that $g \in N_{\theta \circ \theta-1}$. Hence $N \subseteq N_{\theta \circ \theta-1}$.

As a consequence of these lemmas, we have

TheOREM 4.5. Let $(G, \mathscr{X}, \mathscr{Y})$ be a McAlister triple, let $S=P(G, \mathscr{X}, \mathscr{Y})$ and let $\rho$ be an $E$ unitary idempotent-separating congruence on $S . A$ relation $\kappa$ is defined on $\mathscr{X}$ by the rule that

$$
(A, B) \in \kappa \Leftrightarrow B=n A \text { for some } n \in N_{\rho} .
$$

Then $\kappa$ is a $T$-congruence on $\mathscr{X}$ such that $\kappa \cap(\mathscr{Y} \times \mathscr{Y})=1_{\mathscr{Y}}$ and $N_{p} \subseteq \operatorname{ker}(G, \mathscr{X} / \kappa, \mathscr{Y} / \kappa)$. Further, $S / \rho \cong P\left(G / N_{\rho}, \mathscr{X} / \kappa, \mathscr{Y} / \kappa\right)$

Proof. Since $\rho$ is $E$-unitary, $M_{p}=N_{p}$, by Lemma 4.2. Let us take $N=N_{p}$ in Lemma 4.4. Then $\kappa$ is a $T$-congruence on $\mathscr{X}, \kappa \cap(\mathscr{Y} \times \mathscr{Y})=l_{\mathscr{y}}$ and $N_{\rho} \subseteq \operatorname{ker}(G, \mathscr{X} / \kappa, \mathscr{Y} / \kappa)$. Now define $\theta$ as in Lemma 4.4. Since $\theta$ is a surjective homomorphism, $S / \theta \circ \theta^{-1} \cong P\left(G / N_{\rho}, \mathscr{X} / \kappa, \mathscr{Y} / \kappa\right)$. Also, $N_{\theta \circ \theta^{-1}}=N_{\rho}$. Hence, by Lemma $4.3, \theta \circ \theta^{-1}=\rho$.

5. An application. In this section we apply the results of the previous two sections to obtain a proof of McAlister's Theorems A and B stated in $\S 2$.

First, we have the following lemma, which combines the results of Theorems 3.2 and 4.5 .

LeMma 5.1. Let $S$ be a P-semigroup and let $\rho$ be a congruence on $S$. Then there exists a $P$-semigroup $T$ such that $T$ is isomorphic to a quotient of $S$ by an idempotent-determined con- 
gruence and $S / \rho \cong T / \tau$, where $\tau$ is an idempotent-separating congruence on $T$. Furthermore, if $\rho$ is E-unitary then $S / \rho$ is isomorphic to a P-semigroup.

Proof. Let $S=P(G, \mathscr{X}, \mathscr{Y})$ for some McAlister triple $(G, \mathscr{X}, \mathscr{Y})$. Write $\pi=\rho \cap(E \times E)$, where $E$ denotes the semilattice of $S$. Then $\pi$ is a normal equivalence on $E$ and $S / \rho \cong$ $\left(S / \pi^{*}\right) /\left(\rho / \pi^{*}\right)$. But $\pi^{*}$ is an idempotent-determined congruence on $S$ and $S / \pi^{*}$ is isomorphic to a $P$-semigroup $T$, say, by Theorem 3.2. Also $\rho / \pi^{*}$ is idempotent-separating. Hence $S / \rho \cong T / \tau$, for some idempotent-separating congruence $\tau$ on $T$. Now suppose, in addition, that $\rho$ is $E$-unitary. Then $\tau$ is $E$-unitary and so $T / \tau$ is isomorphic to a $P$-semigroup, by Theorem 4.5.

Next, we review some properties of free inverse semigroups. The structure of such semigroups was determined by Scheiblich $[18,19]$; different approaches have been given, independently, by Munn $[10,11]$ and Preston [14].

Let $Z$ be a nonempty set and let $G=\mathscr{F}_{Z}$, the free group on $Z$. Following Scheiblich, we define a partial ordering $\leqslant$ of $G$ by the rule that, for all $a, b \in G, a \leqslant b$ if and only if, when $a$ and $b$ are written in reduced form, $a$ is an initial segment of $b$. The identity of $G$ will be denoted by 1 and we make the convention that $1 \leqslant a$ for all $a \in G$. For $a \in G$ we write $\bar{a}=\{x \in G: x \leqslant a\}$ and for $A \subseteq G$ we write $\bar{A}=\bigcup_{a \in A} \bar{a}$. It is easily verified that a subset $A$ of $G$ which contains 1 is convex under $\leqslant$ if and only if $\bar{A}=A$.

We now describe Scheiblich's construction for the free inverse semigroup on $Z$. Let $\mathscr{Y}$ denote the set of all finite convex subsets of $G$ containing 1 and at least one other element. Write

$$
F=\{(A, g) \in \mathscr{Y} \times G: g \in A\} .
$$

For $g \in G$ and any nonempty subset $B$ of $A$ let $g B$ denote $\{g b: b \in B\}$. It can be shown that if $(A, g),(B, h) \in F$ then $A \cup g B \in \mathscr{Y}$. Hence we can define a multiplication on $F$ by the rule:

$$
(A, g)(B, h)=(A \cup g B, g h) .
$$

Under this multiplication $F$ is the free inverse semigroup $\mathscr{F}_{Z}$ on $Z[18,19]$.

A subset $M$ of an inverse semigroup $S$ is termed a set of generators of $S$ if and only if every element of $S$ can be expressed as a product of elements of the form $x^{\varepsilon}(x \in M, \varepsilon \in\{1,-1\})$. Let $K=\left\{k_{z}: z \in Z\right\}$, where $k_{z}=(\{1, z\}, z)$. Then it can be shown that $K$ generates $F$. Moreover, if $\phi$ is an arbitrary mapping from $K$ to an inverse semigroup $S$ then $\phi$ can be extended uniquely to a homomorphism from $F$ to $S$ (and this is surjective if $K \phi$ generates $S$ ). We express this condition by saying that $K$ generates $F$ freely. Free generators of free inverse semigroups have been studied by Reilly [15].

McAlister and McFadden [7] observed that $F$ can be expressed as a $P$-semigroup. We outline a proof. First, let us write

$$
\mathscr{X}=\{g A: g \in G, A \in \mathscr{Y}\} .
$$

This is a subset of the power set of $G$ and we define a partial ordering $\leqslant$ of it by the rule:

$$
C \leqslant D \Leftrightarrow C \supseteq D \text {. }
$$


Next, we note that the mapping $(g, C) \mapsto g C$ is an action of $G$ on $\mathscr{X}$ by order automorphisms. It can then be shown that $(G, \mathscr{X}, \mathscr{Y})$ is a McAlister triple and that

$$
g^{-1} A \in \mathscr{Y} \Leftrightarrow g \in A \quad(g \in G, A \in \mathscr{Y}) .
$$

Let $S=P(G, \mathscr{X}, \mathscr{Y})$. In view of $\left(^{*}\right)$ the elements of $S$ coincide with those of $F$. Furthermore, the multiplication of elements of $S$ agrees with that in $F$. Thus the semigroups $S$ and $F$ are identical.

Let $\tau$ denote the least group congruence on $F$. Then, from (T5) and (2.2), $F / \tau \cong G \cong$ $\mathscr{F} \mathscr{G}_{Z}$. Moreover, with $K$ as above, $K \tau \hbar=\left\{k_{z} \tau: z \in Z\right\}$ is a set of free generators of $F / \tau$.

Evidently, $F$ does not have an identity. In fact, $\mathscr{Y}$ (which is isomorphic to the semilattice of $F$ ) has $2|Z|$ maximal elements if $Z$ is finite $(|Z|$ if $Z$ is infinite). These are the elements $\{1, z\}$ and $\left\{1, z^{-1}\right\}(z \in Z)$. A straightforward argument, making use of (2.2) and (2.3), shows that $F^{1}$ is an $F$-inverse semigroup. From this it can be deduced (McAlister, unpublished) that $\mathscr{X}$ is a lower semilattice. Alternatively, for this last result we may proceed directly as follows. Consider two typical elements $g A, h B \in \mathscr{X}(g, h \in G ; A, B \in \mathscr{Y})$. Suppose that, in reduced form, $g=k g_{1}, h=k h_{1}$, where $g_{1}, h_{1}$ have no common initial segment except 1 . Then it may be checked that $g A \cup h B \cup k \bar{g}_{1} \cup k h_{1}$ is the greatest lower bound of $g A$ and $g B$ in $\mathscr{X}$.

Using the fact that a free inverse semigroup can be expressed as a $P$-semigroup we now derive McAlister's Theorems A and B.

THEOREM 5.2 (McAlister). Let $S$ be an inverse semigroup.

(i) There exists a P-semigroup $T$ and an idempotent-separating congruence $\tau$ on $T$ such that $S \cong T / \tau$.

(ii) If $S$ is E-unitary then $S$ is isomorphic to a P-semigroup.

Proof. Let $M$ be a set of generators of $S$, let $Z$ be a set of the same cardinal as $M$ and let $F=\mathscr{G} \mathscr{I}_{\mathrm{Z}}$. Then there exists a surjective homomorphism $\theta: F \rightarrow S$. Thus $S \cong F / 0 \circ \theta^{-1}$. But $F$ can be expressed as a $P$-semigroup. The result now follows immediately from Lemma 5.1. I

By a quasi-free inverse semigroup we shall mean a semigroup which is isomorphic to a quotient of a free inverse semigroup by an idempotent-determined congruence. We end this section with a theorem concerning such semigroups.

THEOREM 5.3. (i) Every inverse semigroup is isomorphic to the quotient of a quasi-free inverse semigroup by an idempotent-separating congruence.

(ii) Let $S$ be a quasi-free inverse semigroup. Then there exists a McAlister triple $\left(G, \mathscr{X}^{\prime}, \mathscr{Y}^{\prime}\right)$, where $G$ is a free group and $\mathscr{X}^{\prime}$ is a lower semilatice, such that $S \cong P\left(G, \mathscr{X}^{\prime}, \mathscr{Y}^{\prime}\right)$. Moreover, $S^{1}$ is an F-inverse semigroup.

(iii) Let $S$ be an inverse semigroup with least group congruence $\sigma$. Then $S$ is quasi-free if and only if (a) $S / \sigma$ is a free group and (b) $S$ has a set $M$ of generators such that $\sigma$ a $\left.\right|_{M}$ is injective and $M \sigma$ h generates $S / \sigma$ freely.

Proof. (i) This follows from Lemma 5.1 in the same way as does Theorem 5.2 (i).

(ii) Suppose, without loss of generality, that $S=F / \rho$, where $F$ is a free inverse semigroup and $\rho$ is an idempotent-determined congruence on $F$. Now $F=P(G, \mathscr{X}, \mathscr{Y})$ for some McAlister 
triple $(G, \mathscr{X}, \mathscr{Y})$, with $G$ a free group and $\mathscr{X}$ a lower semilattice. Hence, by Theorem 3.2 , there exists a $T$-congruence $\kappa$ on $\mathscr{X}$ such that $F / \rho \cong P\left(G, \mathscr{X}^{\prime}, \mathscr{Y}^{\prime}\right)$, where $\mathscr{X}^{\prime}=\mathscr{X} / \kappa$ and $\mathscr{Y}^{\prime}=\mathscr{Y} / \kappa$; further, since $\mathscr{X}$ is a lower semilattice, so also is $\mathscr{X}^{\prime}$. Moreover, $F^{1}$ is an $F$-inverse semigroup and so, by Lemma $1.7, S^{1}$ is an $F$-inverse semigroup.

(iii) Suppose that $S$ is quasi-free. We shall assume that $S=F / \rho$, where $F=\mathscr{F}_{Z}$ for some $Z$ and $\rho$ is an idempotent-determined congruence on $F$. Let $k_{z}$ denote $(\{1, z\}, z)$ for all $z \in Z$ and let $K=\left\{k_{z}: z \in Z\right\}$. Write $M=K \rho \natural=\left\{k_{z} \rho: z \in Z\right\}$. Then $M$ generates $S$, since $K$ generates $F$. Let $\tau$ denote the least group congruence on $F$. Then, by Lemma 1.7, $\rho \subseteq \tau$ and so, by (1.5), $\tau / \rho=\sigma$, the least group congruence on $S$. Now there exists a McAlister triple $(G, \mathscr{X}, \mathscr{Y})$, with $G=\mathscr{F} \mathscr{G}_{Z}$, such that $F=P(G, \mathscr{X}, \mathscr{Y})$. Also, for all $(A, g),(B, h) \in F$,

$$
\begin{aligned}
(A, g) \rho \sigma \natural=(B, h) \rho \sigma \natural & \Leftrightarrow((A, g) \rho,(B, h) \rho) \in \sigma \\
& \Leftrightarrow((A, g),(B, h)) \in \tau \\
& \Leftrightarrow g=h, \text { by }(2.2) .
\end{aligned}
$$

Hence we can define an injective mapping $\theta: G \rightarrow S / \sigma$ by the rule that $g \theta=(A, g) \rho \sigma$ h for some (any) $A \in \mathscr{Y}$ such that $(A, g) \in F$. Clearly $\theta$ is also a surjective homomorphism and so an isomorphism. Thus $S / \sigma$ is free. Also, since $z \theta=k_{z} \rho \sigma$ h for all $z \in Z$ and $\theta$ is injective, it follows that $\left.\sigma \curvearrowleft\right|_{M}$ is injective. Further, $Z \theta=M \sigma$. Hence, since $Z$ generates $G$ freely, $M \sigma$ h generates $S / \sigma$ freely.

Conversely, suppose that (a) $S / \sigma$ is free and (b) $S$ has a set $M$ of generators such that $\sigma$ h $\left.\right|_{M}$ is injective and $M \sigma$ t generates $S / \sigma$ freely. Choose a set $Z$ of the same cardinal as $M$ and let $\phi: Z \rightarrow M$ be a bijection. Write $F=\mathscr{F} \mathscr{I}_{Z}$ and let $k_{z}(z \in Z), K$ be defined as before. Then $K$ generates $F$ and the bijection $k_{z} \mapsto z \phi$ from $K$ to $M$ can be extended to a (unique) homomorphism $\theta: F \rightarrow S$. Since $M$ generates $S$ it follows that $\theta$ is surjective. Thus $S \cong F / \theta \circ \theta^{-1}$. Let $\tau$ denote the least group congruence on $F$. By Lemma 1.7, to show that $S$ is quasi-free it suffices to prove that $\theta \circ \theta^{-1} \subseteq \tau$.

Since $\theta \sigma$ is a homomorphism from $F$ to $S / \sigma$ and $\tau$ is the least group congruence on $F$, there exists a surjective homomorphism $\psi: F / \tau \rightarrow S / \sigma$ such that $\theta \sigma \hbar=\tau \hbar \psi$. Now $\left.\theta\right|_{R}$ is a bijection from $K$ to $M$ and, by hypothesis, $\sigma$ ต $\left.\right|_{M}$ is a bijection from $M$ to $M \sigma$. Thus $\left.\tau \dot{q} \psi\right|_{\mathrm{R}}$ is a bijection from $K$ to $M \sigma \hbar$. But $\tau$ h $\left.\right|_{K}$ is a bijection from $K$ to $K \tau \hbar$, by (2.2). Hence $\psi$ induces

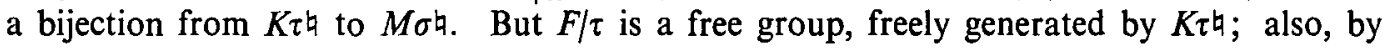
hypothesis, $S / \sigma$ is a free group, freely generated by $M \sigma$. Hence $\psi$ must be an isomorphism. Consequently,

$$
\theta \circ \theta^{-1} \subseteq(\theta \sigma \natural) \circ(\theta \sigma \natural)^{-1}=(\tau \natural \psi) \circ(\tau \natural \psi)^{-1}=\tau \natural \circ(\tau \natural)^{-1}=\tau,
$$

and so $S$ is quasi-free.

REMARK. It is possible to construct a McAlister triple whose middle component is not a lower semilattice. Let $\left(G^{\prime}, \mathscr{X}^{\prime}, \mathscr{Y}^{\prime}\right)$ be one such and let $S=P\left(G^{\prime}, \mathscr{X}^{\prime}, \mathscr{Y}^{\prime}\right)$. By Theorem 5.3 (i) and (ii), $S \cong T / \tau$, where $T=P(G, \mathscr{X}, \mathscr{Y})$ for some McAlister triple $(G, \mathscr{X}, \mathscr{Y})$ in which $\mathscr{X}$ is a lower semilattice, and where $\tau$ is an idempotent-separating congruence on $T$. Then, by Theorem 4.5 and the isomorphism theorem, there exists a $T$-congruence $\kappa$ on $\mathscr{X}$ such that $\mathscr{X}^{\prime}$ is order isomorphic to $\mathscr{X} / \kappa$. Hence, in this case, $\kappa$ is not a semilattice congruence on $\mathscr{X}$. 
6. The lattice of E-unitary congruences. We conclude with a brief discussion of properties of the set of all $E$-unitary congruences on an arbitrary inverse semigroup.

THEOREM 6.1. Let $S$ be an inverse semigroup with semilattice $E$ and least group congruence $\sigma$. Let $\Lambda(S)$ denote the lattice of congruences on $S$ and let $\Lambda^{*}$ denote the subset of $\Lambda(S)$ consisting of all E-unitary congruences.

(i) $\Lambda^{*}$ is a complete lattice with respect to inclusion.

(ii) The least element $\lambda$ of $\Lambda^{*}$ is contained in $\sigma$ and $\{\rho \in \Lambda(S): \lambda \subseteq \rho \subseteq \sigma\}$ is a complete sublattice of $\Lambda^{*}$.

(iii) If $\alpha, \beta \in \Lambda^{*}$ are such that $\alpha \subseteq \beta$ and $\alpha \cap(E \times E)=\beta \cap(E \times E)$ then $\left\{\gamma \in \Lambda^{*}: \alpha \subseteq \gamma \subseteq \beta\right\}$ is a complete modular sublattice of $\Lambda^{*}$.

Proof. (i) We first note that $S \times S \in \Lambda^{*}$. Let $\left(\rho_{i}\right)_{i \in I}$ be a nonempty family of elements of $\Lambda^{*}$ and let $\rho$ denote $\bigcap_{i \in I} \rho_{i}$. We show that $\rho \in \Lambda^{*}$. Let $a, b \in S$ be such that $(a b, b) \in \rho$ and $\left(b, b^{2}\right) \in \rho$. Then, for all $i \in I,(a b, b) \in \rho_{i}$ and $\left(b, b^{2}\right) \in \rho_{i}$, from which it follows that $\left(a, a^{2}\right) \in \rho_{i}$. Hence $\left(a, a^{2}\right) \in \rho$ and so $\rho \in \Lambda^{*}$. Thus $\left(\rho_{i}\right)_{i \in I}$ has a greatest lower bound in $\Lambda^{*}$, namely $\rho$. Now, since $\Lambda^{*}$ has a greatest element, the subset $\left\{\tau \in \Lambda^{*}: \tau \supseteq \rho_{i}\right.$ for all $\left.i \in I\right\}$ is nonempty and the intersection $\hat{\rho}$ of all its members is again in $\Lambda^{*}$. Hence $\left(\rho_{i}\right)_{i \in I}$ has a least upper bound in $\Lambda^{*}$, namely $\hat{\rho}$. We have thus shown that $\Lambda^{*}$ is a complete lattice with respect to inclusion (and also a complete lower subsemilattice of $\Lambda(S)$ ). In particular, as noted by $O^{\prime}$ Carroll [12, Theorem 5], $\Lambda^{*}$ has a least element $\lambda$.

(ii) Every group congruence on $S$ lies in $\Lambda^{*}$ and so $\lambda \subseteq \sigma$. Write $M=\{\rho \in \Lambda(S)$ : $\lambda \subseteq \rho \subseteq \sigma\}$. Evidently $M$ is a complete sublattice of $\Lambda(S)$. We show that $M \subseteq \Lambda^{*}$. Let $\rho \in M$. Then $\rho / \lambda \subseteq \sigma / \lambda$. But $\sigma / \lambda$ is the least group congruence on $S / \lambda$, by (1.5). Hence, by Lemma $1.7, \rho / \lambda$ is an $E$-unitary congruence on $S / \lambda$. Now $S / \rho \cong(S / \lambda) /(\rho / \lambda)$. Hence $S / \rho$ is $E$-unitary and so $\rho \in \Lambda^{*}$. Thus $M \subseteq \Lambda^{*}$. Since $M$ is a complete sublattice of $\Lambda(S)$ it must therefore also be a complete sublattice of $\Lambda^{*}$. (It is easy to see that $M$ is isomorphic to the lattice of all idempotent-determined congruences on $S / \lambda$.)

(iii) Let $\alpha, \beta \in \Lambda^{*}$ be such that $\alpha \subseteq \beta$ and $\alpha \cap(E \times E)=\beta \cap(E \times E)$. We apply the results of $\S 4$ to the $E$-unitary inverse semigroup $S / \alpha$. By Theorem 5.2 (ii), we may, without loss of generality, take $S / \alpha=P(G, \mathscr{X}, \mathscr{Y})$ for some McAlister triple $(G, \mathscr{X}, \mathscr{Y})$. For any idempotentseparating congruence $\xi$ on $S / \alpha$ let subsets $M_{\xi}, N_{\xi}$ of $G$ be defined as in $\S 4$. In particular, by Lemma $4.1, N_{\xi}$ is a normal subgroup of $G$ and, by Lemma 4.2 , if $\xi$ is $E$-unitary then $M_{\xi}=N_{\xi}$. Write $\rho=\beta / \alpha$. Then $\rho$ is an $E$-unitary idempotent-separating congruence on $S / \alpha$. Now $\gamma \mapsto \gamma / \alpha$ is an order isomorphism from $\left\{\gamma \in \Lambda^{*}: \alpha \subseteq \gamma \subseteq \beta\right\}$ to the set $\Gamma_{\rho}$ of all $E$-unitary (idempotent-separating) congruences on $S / \alpha$ contained in $\rho$. Let $\Sigma_{\rho}$ denote the set of all normal subgroups of $G$ contained in $M_{\rho}$. Since $\rho$ is $E$-unitary, $M_{\rho}$ is a normal subgroup of $G$ and so $\Sigma_{\rho}$ is a complete modular lattice with respect to inclusion. Now consider the mapping $\phi: \Gamma_{p} \rightarrow \Sigma_{\rho}$ defined by $\xi \phi=N_{\xi}$. By Lemma 4.4 (iii), $\phi$ is surjective and so, by Lemma $4.3, \phi$ is an order isomorphism.

The following example shows that $\Lambda^{*}$ need not be a sublattice of $\Lambda(S)$. Let $G$ be a nontrivial group and let $H$ denote the direct product $G \times G$. Take $S$ to be the semilattice of groups 
$G \cup H$, with $H$ as least ideal and multiplication determined by the structure homomorphism $\phi: G \rightarrow H$ defined by $g \phi=(g, g)$ [1, Theorem 4.11]. It is easily seen that $S$ is an $E$-unitary inverse semigroup and that the relations

$$
\begin{aligned}
& \rho_{1}=\{((a, c),(b, c)) \in H \times H\} \cup i_{s}, \\
& \rho_{2}=\{((a, b),(a, c)) \in H \times H\} \cup i_{s}
\end{aligned}
$$

are $E$-unitary idempotent-separating congruences on $S$. Let $\rho_{1} \vee \rho_{2}$ denote the join of $\rho_{1}$ and $\rho_{2}$ in $\Lambda(S)$. Then $\rho_{1} \vee \rho_{2}=(H \times H) \cup l_{s}$. Hence $S /\left(\rho_{1} \vee \rho_{2}\right) \cong G^{0}$ and so, since $G$ is nontrivial, $\rho_{1} \vee \rho_{2}$ is not $E$-unitary. Thus, in this case, $\Lambda^{*}$ is not a sublattice of $\Lambda(S)$.

Now let us again assume the hypotheses of Theorem 6.1, with $S$ an arbitrary inverse semigroup. Let $\pi$ be a normal equivalence on $E$ and let $\lambda \vee \pi^{*}$ denote the join of $\lambda$ and $\pi^{*}$ in $\Lambda(S)$. Since $\lambda \subseteq \lambda \vee \pi^{*} \subseteq \sigma$ it follows from part (ii) of the theorem that $\lambda \vee \pi^{*} \in \Lambda^{*}$. From this we deduce that, for any $\theta$-class $\Theta$ of $\Lambda(S)$ (see (1.3)),

$$
\Theta \cap \Lambda^{*} \neq \emptyset \Leftrightarrow \lambda \theta \leqslant \Theta,
$$

where $\leqslant$ denotes the partial ordering of the quotient lattice $\Lambda(S) / \theta$.

Finally, suppose that in part (iii) of the theorem we no longer assume that $\beta$ is $E$-unitary. Then, with the notation of the proof, we again have an order isomorphism from $\left\{\gamma \in \Lambda^{*}\right.$ : $\alpha \subseteq \gamma \subseteq \beta\}$ to $\Sigma_{\rho}$, the set of normal subgroups of $G$ contained in $M_{\rho}$ and ordered by inclusion; however, $M_{\rho}$ need no longer be a subgroup of $G$. A straightforward application of Zorn's lemma shows that $\left\{\gamma \in \Lambda^{*}: \alpha \subseteq \gamma \subseteq \beta\right\}$ has a maximal element and that every element of this set is contained in a maximal element. These remarks apply, in particular, to the case in which $\rho(=\beta / \alpha)$ is the greatest idempotent-separating congruence on $S / \alpha$.

\section{REFERENCES}

1. A. H. Cliftord and G. B. Preston, The algebraic theory of semigroups, Math. Surveys of the Amer. Math. Soc. 7 (Providence, R.I., 1961 (vol. 1) and 1967 (vol. 2)).

2. D. G. Green, Extensions of a semilattice by an inverse semigroup, Bull. Austral. Math. Soc. 9 (1973), 21-31.

3. J. M. Howie, The maximum idempotent-separating congruence on an inverse semigroup, Proc. Edinburgh Math. Soc. (2) 14 (1964), 71-79.

4. D. B. McAlister, Groups, semilattices and inverse semigroups, Trans. Amer. Math. Soc. 192 (1974), 227-244.

5. D. B. McAlister, Groups, semilattices and inverse semigroups II, Trans. Amer. Math. Soc. 196 (1974), 351-370.

6. D. B. McAlister and R. McFadden, Zig-zag representations and inverse semigroups, J. Algebra 32 (1974), 178-206. 652-666

7. R. McFadden and L. O'Carroll, F-inverse semigroups, Proc. London Math. Soc. (3) 22 (1971),

8. W. D. Munn, A class of irreducible matrix representations of an arbitrary inverse semigroup, Proc. Glasgow Math. Assoc. 5 (1961), 41-48. $157-170$.

9. W. D. Munn, Fundamental inverse semigroups, Quarterly J. Math. Oxford (2) 21 (1970), 
10. W. D. Munn, Free inverse semigroups, Semigroup Forum 5 (1973), 262-269. (Research announcement.)

11. W. D. Munn, Free inverse semigroups, Proc. London Math. Soc. (3) 29 (1974), 385-404.

12. L. O'Carroll, Reduced inverse semigroups, Semigroup Forum 8 (1974), 270-276. (Research announcement.)

13. L. O'Carroll, Reduced inverse and partially ordered semigroups, J. London Math. Soc. (2) 9 (1974), 293-301.

14. G. B. Preston, Free inverse semigroups, J. Austral. Math. Soc. 16 (1973), 443-453.

15. N. R. Reilly, Free generators of free inverse semigroups, Bull. Austral. Math. Soc. 7 (1972), $407-424$.

16. N. R. Reilly, E-unitary inverse semigroups, Math. Dept. Research Report, Simon Fraser University (1975).

17. N. R. Reilly and H. E. Scheiblich, Congruences on regular semigroups, Pacific J. Math. 23 (1967), 349-360.

18. H. E. Scheiblich, Free inverse semigroups, Semigroup Forum 4 (1972), 351-359. (Research announcement.)

19. H. E. Scheiblich, Free inverse semigroups, Proc. Amer. Math. Soc. 38 (1973), 1-7.

20. T. Saitô, Proper ordered inverse semigroups, Pacific J. Math. 15 (1965), 649-666.

SiMON Fraser UNIVERSITY

UNIVERSITY OF GLASGOW

Burnaby 2, B.C., Canada 